\title{
The STAUT-Reader, making general-purpose texts accessible to deaf.
}

\author{
Aníbal C. de Oliveira ${ }^{1}$, Francisco C.M.B. Oliveira ${ }^{1}$, Eudenia M. Barros ${ }^{1}$, \\ Adriano T. de Freitas ${ }^{3}$, Thiago A.C. de Araujo ${ }^{1}$, Lidiane C. Silva ${ }^{1}$ \\ ${ }^{1}$ Departamento de Computação - Universidade Estadual do Ceará (UECE) \\ Fortaleza - CE - Brazil \\ ${ }^{2}$ Inst. de Filosofia e Ciênc. Humanas - Universidade Estadual de Campinas (UNICAMP) \\ São Paulo - SP - Brazil \\ ${ }^{3}$ Departamento de Computação - Inst. Federal do Ceará (IFCE) \\ Maracanaú - CE - Brazil \\ \{hanibal.ce80,fran.mb.oliveira, eudenia.magalhaes\}@gmail.com \\ \{adrianoblue, thiago.ac.araujo, lidcastro\}@gmail.com
}

\begin{abstract}
Deaf students have difficulty in reading texts written in languages whose mode is oral-auditory, such as Portuguese. As users of a signed language, it is harder for them to obtain information contained in books, magazines and newspapers. For this purpose, we have built the STAUT-Reader, an electronic reader, designed to help deaf to read texts written in Portuguese. The tool uses a Rule-Based Machine Translation system to produce output texts in a written representation of Libras, the Brazilian Sign Language. To investigate our proposal, we conducted an experiment using a counterbalanced within-subjects design to evaluate the reading performance of deaf readers using our tool. The results showed to be promising, especially for non-oralized deaf.
\end{abstract}

\section{Introduction}

Brazilian deaf readers have difficulty to read and write in Portuguese [Luccas et al. 2012]. Their natural language (L1) is the Brazilian Sign Language (Libras), a visual-spatial language. Thus, the spoken and written Portuguese, whose mode is oral-auditory, appears as a second language (L2), and its acquisition will be made artificially, resembling the learning of a foreign language, with the aggravation of being a language in a different modality [Quadros 1997].

However, the amount of educational content and teaching materials available in Libras are still scarce [Oliveira 2012]. In part, this is due to the high costs involved in producing materials for this audience [Quadros 1997], as well as, the short age of Libras, which was recognized by means of law as an official language, only in 2002 [Brasil 2002].

We believe that technology can be a strategy to overcome this impediment. It helps to reduce barriers in both written and spoken communication, providing accessible tools for teaching and learning [Sassaki 2005]. In addition, since the deaf community is geographically distributed throughout Brazil, providing online content and materials, for instance, helps to reduce the difficulties imposed by distance, enabling the democratization of education [Larreamendy-Joerns and Leinhardt 2006]. 
In this context, this paper presents a prototype of the STAUT-Reader, an electronic reader, which aims to help deaf students to read, comprehend and retain information of texts originally available in Portuguese. The tool translates texts to a written representation of Libras, a textual representation of the signs, also referred as gloss notation [Bungerot et al. 2008]. Our proposal is to create a tool that expands the access to materials that were previously accessible only in Portuguese, thus broadening the range of educational materials available for deaf students.

The STAUT-Reader consists of two main modules: the translation module and the reading module. The translation module is a Rule-Based Machine Translation (RBMT) system, responsible to transform Portuguese texts into its corresponding terms in gloss notation. The reading module is a web-based system where users can select the input texts and then read them translated.

To validate our proposal, we conducted an experiment using a counterbalanced within-subjects design to assess the ability of 20 students on reading texts written in gloss notation. More precisely, we measured the effect of the STAUT-Reader on perceived and actual text difficulty [Leroy et al. 2013]. The perceived difficulty was measured with one metric, a 5-point Likert scale. The actual difficulty was measured with two metrics: 5 multiple-choice questions alongside the text to measure understanding, and 5 multiplechoice questions without the text for learning and information retention.

Concerning the perceived difficulty, all the sentences in gloss notation were better assessed than the ones in Portuguese. On the actual difficulty stage, although there was not found a significant statistical result, the means obtained indicates that there is a trend that might benefit non-oralized readers.

This paper is organized as follows. In Section 2, we present a brief discussion about paper reading versus electronic reading; in Section 3, we show related work; In Section 4, we detail the STAUT-Reader's operation and architecture; In Section 5, we describe the experiment and its results. Finally, in Section 6, we present the conclusions and future works.

\section{Electronic Reading vs Paper Reading}

Recent studies have been devoted to investigate which are the main differences between the contemporary forms of reading: the conventional one held in printed papers, such as books, magazines and newspapers; and the reading using digital tools, such as computers and mobile devices, which are commonly available as e-books and electronic newspapers.

Studies conducted at the University of Stavanger in Norway show that there is a slight disadvantage of digital readers because they allegedly diminish the individual's attention due to its multitasking support in a digital environment [Mangen et al. 2013]. In this case, the study was conducted by comparing two groups - subjects who read printed texts in paper, and subjects who read through a computer screen. The first group was more successful in understanding the texts. The hypotheses raised by researchers, to such results, are related to navigation elements, layout and the direct access to the totality of printed texts, since such settings does not occur in the digital reading scenario.

In contrast, a research conducted by the Harvard-Smithsonian Center for Astrophysics indicates that when comparing the two types of reading among a group of 
adolescents with dyslexia, those who had more limitations to decode phonemes or had less capacity to visual attention, had better success in understanding the texts presented [Schneps et al. 2013] electronically. The team realized that a portable device improved the speed and understanding among the group because of its capability of reformatting pages, which facilitates the conditions of those who are harmed by the temporal dynamics in reading, such as slowness caused by attention deficit and difficulties accessing phonological representations of words or character recognition.

Also, Korat and Shamir [Korat and Shamir 2008] developed a computerized educational book as a supporting tool for the development of preschoolers' emergent literacy. For that, they conducted a research with 149 children from different socioeconomic status. The results showed that with the use of the e-book, children had improvements in their vocabulary, word recognition and phonological awareness level. In addition, Quek, Elglaly and Oliveira[Quek et al. 2013] demonstrate with their e-Reader system for individuals with blindness or severe visual impairment that electronic reading can help in the processing and presentation of information that promotes their engagement in active reading at their own pace and control.

It seams that electronic reading presents an opportunity for researchers and practitioners to present textual information through different interfaces, adaptable to the reader's needs and disabilities. We propose a tool capable of processing texts in Portuguese and presenting them in also a textual form, whose components are aligned, as they would be if signalized in Libras. We hope that this strategy will facilitate understanding and retention of information contained on the texts.

\section{Related Work}

There are many efforts of the scientific community to develop tools that help people with disabilities to have better access to information. However, we found only a few studies on the reading of written texts for deaf, especially for those who use Libras as first language. Below, we present other ongoing research projects related to the reading for the deaf.

The PorSimples Project offers a set of tools designed to perform text adaptations for low literacy readers. It consists of a set of text simplification tools, such as: SIMPLIFICA, which helps authors to create simplified texts; FACILITA, which explores summarization tasks by simplifying web content; and FACILITA Educational, which assists readers with low literacy to perform detailed reading, displaying questions to clarify semantic relations, linking verbs to their arguments, highlighting named entities, associations between the main ideas of texts and running lexical development [Aluísio and Gasperin 2010].

In addition, researches from the PorSimples project, created an extension component, based on the SIMPLIFICA tool, responsible to assist the Brazilian deaf community to access information. As a result, this module rewrites simplified texts, considering the linguistic aspects of sign language in an interlanguage of Libras. To achieve this result, the system performs a two phase process. The first, called analysis, simplifies the text. The second, called transformation, makes changes in sentences to approach as much as possible of the linguistic characteristics of Libras [Santos et al. 2009].

Another tool that translate texts in Portuguese to facilitate understanding of the deaf is the LIBROL. The software recognizes and discards the particularities in Por- 
tuguese that are not found in Libras. With a simple interface, the software displays on the same screen the original text and its outcome transcribed into words, but obeying the rules of the sign language grammar. The system also has a database with words in simplified Portuguese ant their related synonyms that can be used in Libras. The tool allows the user to feed this database from suggestions of translation improvements [Carvalho et al. 2013]. To assess whether the texts converted by LIBROL improve understanding by the deaf, Silva [Silva et al. 2014] measured the understanding of journalistic texts applied to deaf students. The results showed that the understanding of the texts generated by the system were easier than in Portuguese, but more difficult than in Libras.

The PULO (Portuguese-UNL-LIST DeOralize) is a interlingual machine translation system that translates Portuguese to a representation of signs in Libras, called LIST (Libras Script for Translation). The LIST lexeme still under development and consists in words carried with special characters and marks to emulate the structure of Libras. In its approach, Portuguese texts are transformed into an interlingua, and LIST is then generated from the interlingua. The interlanguage used is the Universal Networking Language (UNL).

The papers presented above have similarities with our research. However, with the exception of LIBROL, none of those texts report studies involving actual Deaf readers. Even so, from what is described in its papers, the LIBROL project does not mention some vital information about its research, such as, the number or the profile of the subjects involved. Therefore, it was not possible for us to perform any deeper evaluation of these tools against the benefits for deaf readers. As we shall see, as a result of our experiment, we could find that a portion of our readers, especially those who had less experience with written Portuguese, have obtained a better result using our tool.

\section{The STAUT-Reader}

\subsection{Simplifying Texts For Deaf}

The Text Simplification technique (TS) has been developed to facilitate the access and provide a better understanding of written texts. Its propose is to promote the expansion of knowledge for a greater number of people, including those with low literacy or with some kind of language barrier [Santos et al. 2009].

Therefore, the TS consists in modifying the text structure to reduce its complexity by changing its lexical and syntactic structure. To achieve that, it goes through three different stages: analysis, transformation and regeneration. After the first stage, when the text was examined and a close reading was performed, a restructuring in its sentences is applied. After that, in the regeneration stage, is verified the cohesion between the original and the simplified text, in order to prevent semantic distinctions in the generated result [Siddharthan 2006] [Chandrasekar et al. 1996].

The STAUT-Reader's TS strategy uses the structure of the sentences in Libras. Different from a normal textual simplification approach, it also incorporates some sign language formation rules [Quadros 2003], such as:

- change the phrase from passive to active voice, prevailing the Subject-VerbObject (SVO) order;

- use, if necessary, the OSV or SOV orders for topicalization, which consists in highlighting the main idea of the sentence, and then contextualize it; 
- remove articles, prepositions, conjunctions, and other linking words;

- use of dactylology to express proper nouns, where in such cases, the word is presented letter by letter, separated by dash.

To represent the morphosyntactic structure of Portuguese and Libras, we designed a mechanism of rule definition for the STAUT-Reader. Through this component, Libras interpreters and deaf readers are able create new rules to represent their knowledge about both languages. The STAUT-Reader will then use those transfer rules to translate the texts from one language to another.

\subsection{The tool}

The STAUT-Reader consists of two modules: the translation and the reading module. The translation module is responsible for receiving input sentences written in Portuguese and translating them to Gloss notation. The reading module is responsible for displaying the translations for the users.

\subsubsection{The Translation Module}

The translation module is a transfer-based Rule-Based Machine Translation (RBMT) system, and uses the linguistic structure of both languages involved in the translation process [Bhattacharyya 2015]. Its approach involves linking the input and output sentences structures using a morphosyntactic analyzer, a set of transfer rules and a sentence generator for the target language. For that, this module has three basic components: the morphosyntactic analyzer (MSA), the rules database, and the output sentence generator (OSG).

The morphosyntactic analyzer (MSA) splits the full text in separated sentences, and for each one, creates an intermediate representation that describes its morphological and syntactical structure. To perform the morphosyntactic analysis, the MSA engine uses the PALAVRAS [Bick 2000] parsing-system, available as a free service only for research purposes, through the CG-3 framework [Denmark. 2015]. This parser performs a grammatical analysis in Portuguese sentences, and provides as the output result, the original sentence elements tagged according to its grammatical classification. In addition, to access the GC-3 web service, the MSA uses a set of Java libraries provided by the HtmlUnit framework's API, which allows browsing web pages from a Java code [HtmlUnit 2015]. The Figure 1 presents the result of PALAVRAS analysis of the text "Pedro comeu pizza" (in English, Pedro ate pizza).

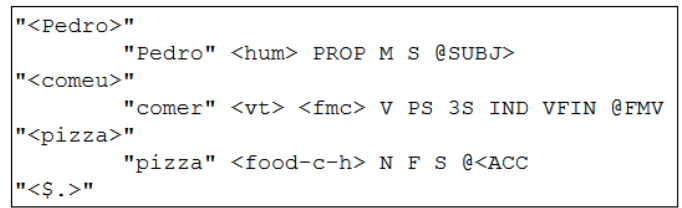

Figure 1. The PALAVRAS analysis.

Notice that the parser PALAVRAS returns a complete analysis of the input text, including the base form of the words (lexeme), tags indicating its lexical category (e.g. PROP for proper nouns, $\mathbf{V}$ for verbs and PS for past simple verbs), and a set syntactic 
function tags (e.g. @SUBJ $>$ indicating the subject of the sentence and $₫<$ ACC for direct object). It also performs a semantic analysis, including tags like $<\mathbf{h u m}>$, to indicate a human name.

Transfer rules are typically structure transforming rules [Bhattacharyya 2015]. Knowing that, we built a mechanism to define and store the rules called rules database (RDB). The rules are described as context-free grammars (CFG). Each rule has its own grammar and represents the syntactical and morphological structure of a Portuguese sentence. The terminals or alphabet $\Sigma$ used to describe each CFG is the set of the morphological and syntactic tags provided by the PALAVRAS. In addition, it uses a set of part-of-speech tags, written with the following delimiters " $<"$ " $>$ ", to represent the nonterminals symbols. The Figure 2 presents an example of a STAUT-Reader simple rule.

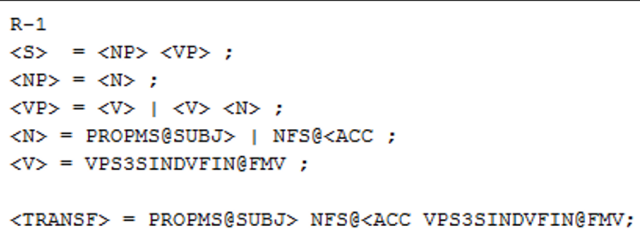

Figure 2. Example of a STAUT-Reader simple rule.

As can be seen, the first line of each rule define its name, for example R-1. Then we have a set of production rules, followed by a blank line, and a final production rule defined by the terminal $<$ TRANSF $>$, a transfer rule. Finally, we have a dashed line indicating the end of the rule. As we will see in the following section, the transfer rule indicates an output structure, corresponding to a translated text for gloss notation. It is also noteworthy that the idea behind building an independent database of rules is that new rules can be added or extended by users.

The output sentence generator (OSG) connects the resulting structures obtained by the MSA to the rules described by the RDB. Given the MSA output structure as input string, the OSG scans all rules defined in the RDB and tries to perform the parsing for each of them. Its parsing algorithm determines if the string is a valid language for at least one grammar defined in the database. In case of success, the OSG uses the transfer rule defined by the terminal $<$ TRANSF $>$ at the end of the accepted rule. This process will be repeated until no other rule is accepted. The Figure 3, shows an example of an input string that was accepted by the second rule and the output result described by the transformation rule.

The OSG implements a LL(1) parser, a top-down parsing algorithm, which produces a leftmost derivation of the input sentence [Louden 1997]. LL(1) parsers can only be constructed for LL(1) grammars, a special case of CFGs that are not ambiguous and not left-recursive [Louden 1997]. Therefore, the OSG eliminates left recursion and performs a left factoring, if necessary, in order to transform the original CFG in a LL(1) equivalent. In case there is not an equivalent LL(1) grammar, the rule will be ignored and an alert message will be sent indicating that it needs to be rewritten. 


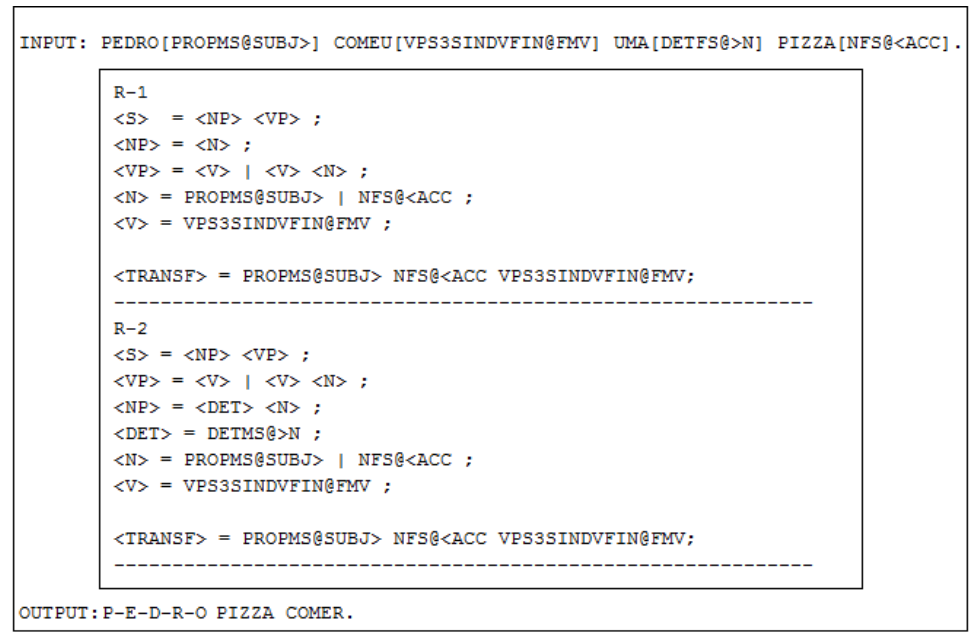

Figure 3. The OSG output

\subsection{The Reading Module}

In Figure 4 we present the reading module of the STAUT-Reader. This is where users can read texts in gloss notation. This module is still under development, since we continue to investigate new features to use along with gloss notation.

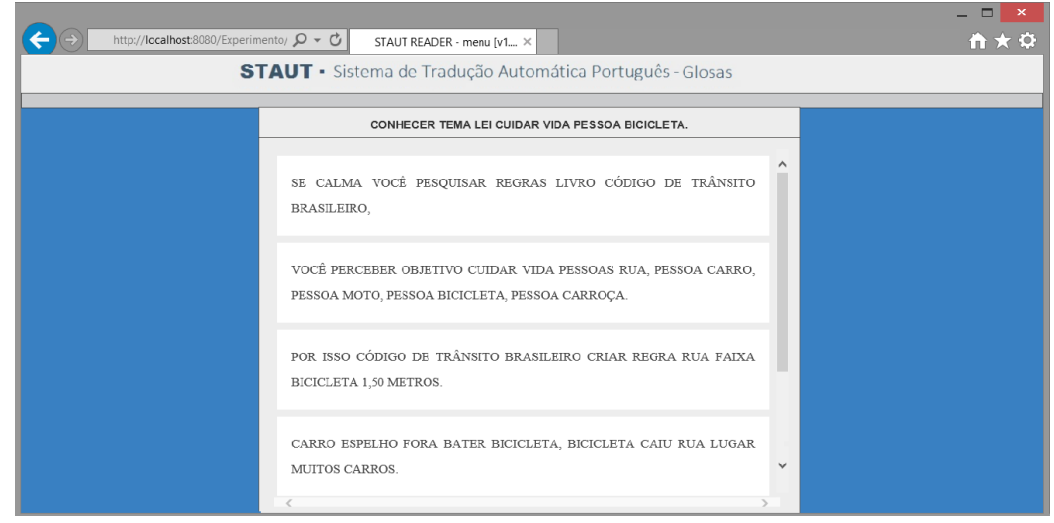

Figure 4. The reading panel

\section{The Experiment}

\subsection{The Experimental Design}

The experiment was conducted with a counterbalanced within-subjects design. The sample consisted of 20 students with the following profiles: 12 female and 8 male; 8 high school graduate and 12 college graduate; and 15 oralized and 5 non-oralized. According to Torres [Torres et al. 2007], oralized deaf are those who have developed more skills in a oral-auditory language, compared to those non-oralized, whose verbal skills are quantitatively and qualitatively inferior.

The experiment consisted of three different stages. The participants were randomly assigned in two groups: A and B. For members from group A, the even-numbered texts were presented in gloss notation, and the odd-numbered texts were presented in Portuguese. The B group members had access to the same texts in the opposite version. 
In the first stage, each participant evaluated 10 sentences ( 5 in Portuguese and 5 in gloss notation) using a 5-point Likert scale options. Our goal was to assess the perceived difficulty of the texts by the subjects. In the second stage, participants were asked to read two different texts and answer a set of 5 multiple-choice questions for each text. The texts remained available for consultation, as participants responded. The objective was to measure the reading difficulty through the understanding and interpretation of texts. Finally, in the third stage, participants also had to answer five multiple-choice questions of two different texts, but they did not have access to the texts while answering the questions. Here, the aim was to measure the learning and the information retention.

\subsection{The Results}

In Stage 1, where we evaluate the perceived difficulty of the texts using a 5-point Likert scale, all texts presented in gloss notation were better assessed than the versions in Portuguese. In Stage 2 and 3, we evaluated the number of correct answers. We ran one-way Anova at a 95\% confidence interval on the data collected, and found the following means presented on Table 1 and Table 2.

Table 1. Stage 2 - Means

\begin{tabular}{|c|c|c|c|c|c|}
\hline Level & Number & Mean & Std Error & Lower 95\% & Upper 95\% \\
\hline Non-Oralized Control & 5 & 1.600 & 0.562 & 0.459 & 2.740 \\
\hline Non-Oralized STAUT & 5 & 2.200 & 0.562 & 1.059 & 3.340 \\
\hline Oralized Control & 15 & 2.733 & 0.324 & 2.074 & 3.391 \\
\hline Oralized STAUT & 15 & 2.000 & 0.324 & 1.341 & 2.658 \\
\hline
\end{tabular}

Table 2. Stage 3 - Means

\begin{tabular}{|c|c|c|c|c|c|}
\hline Level & Number & Mean & Std Error & Lower 95\% & Upper 95\% \\
\hline Non-Oralized Control & 5 & 1.000 & 0.482 & 0.021 & 1.978 \\
\hline Non-Oralized STAUT & 5 & 1.600 & 0.482 & 0.621 & 2.578 \\
\hline Oralized Control & 15 & 1.266 & 0.278 & 0.701 & 1.831 \\
\hline Oralized STAUT & 15 & 1.466 & 0.278 & 0.901 & 2.031 \\
\hline
\end{tabular}

Although there is not a significant statistical result in Stage $2\left(F_{3,40}=0.2633, n s\right)$ and Stage $3\left(F_{3,40}=0.7869, n s\right)$, there is a trend that might benefit non-oralized readers as we can see in the Figure 5.

\section{Conclusion and Future Works}

Creating opportunities for the deaf to access, comprehend and retain information available in texts written in Portuguese is a still an open challenge. We've seen that electronic reading, while not the preferred reading way of reading among people without disabilities, presents the opportunity to render texts in ways that might favor the deaf. We discussed how previous works, armed with natural language processing techniques, fell short in assessing their tools with the deaf. We presented our STAUT-Reader and thoroughly assessed its impacts on activities related to reading, interpreting and retaining information available texts. We've shown that STAUT-Reader seems to create more learning opportunities for the non-oralized deaf than for the oralized deaf. The results seems promising and call for further investigation. The experimental procedure should be repeated with a larger group of deaf students. Furthermore, we are proposing a longitudinal study to investigate the impacts of better understanding and retaining in the long term for highschoolers who are deaf. 


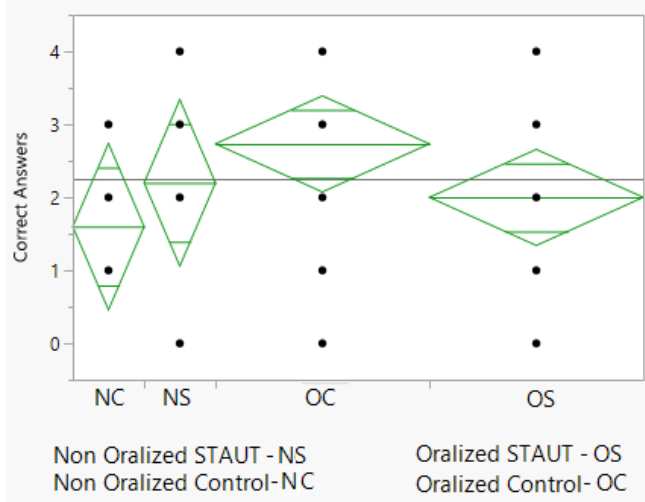

(a) Stage 2 - Understanding

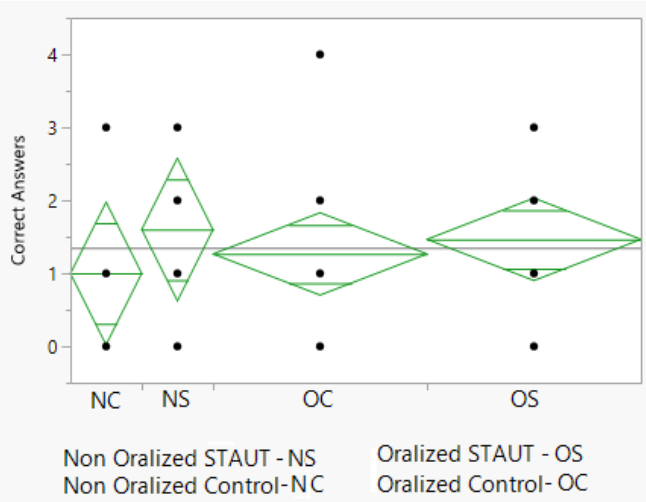

(b) Stage 3 - Learning and Retention

Figure 5. Stage 2 and 3 Correct Answers Means

\section{References}

\section{References}

Aluísio, S. M. and Gasperin, C. (2010). Fostering digital inclusion and accessibility: the porsimples project for simplification of portuguese texts. In Proceedings of the NAACL HLT 2010 Young Investigators Workshop on Computational Approaches to Languages of the Americas, pages 46-53. Association for Computational Linguistics.

Bhattacharyya, P. (2015). Machine Translation. Boca Raton: Taylor \& Francis, A CRC title, part of the Taylor \& Francis imprint, a member of the Taylor \& Franck, Chapman and Hall/CRC.

Bick, E. (2000). The parsing system" Palavras": Automatic grammatical analysis of Portuguese in a constraint grammar framework. Aarhus Universitetsforlag.

Brasil (April,24th 2002). About brazilian sign language (libras) and other provisions. brazilian law no. 10,436. Diario Oficial da Republica Federativa do Brasil - Brasilia, DF - Brasil.

Bungerot, J., Stein, D., Dreuw, P., Ney, H., Morrissey, S., Way, A., and van Zijl, L. (May,28th 2008). The atis sign language corpus. LREC 2008 - Sixth International Conference on Language Resources and Evaluation.

Carvalho, R. S., Brito, J. O., Rodrigues, J. P., Silva, I. Q., Matos, P. F., and de Oliveira, C. R. S. (2013). Librol: Software tradutor de português para libras. CSBC 2013 - XXXIII

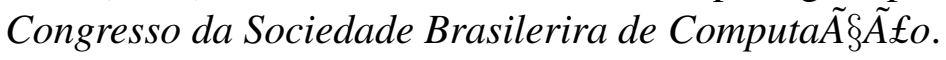

Chandrasekar, R., Doran, C., and Srinivas, B. (1996). Motivations and methods for text simplification. In Proceedings of the 16th conference on Computational linguisticsVolume 2, pages 1041-1044. Association for Computational Linguistics.

Denmark., U. O. S. (2015). Visl - constraint grammar. [Online; accessed May-2015].

HtmlUnit (2015). Htmlunit a gui-less browser for java programs. [Online; accessed May2015].

Korat, O. and Shamir, A. (2008). The educational electronic book as a tool for supporting children's emergent literacy in low versus middle ses groups. Computers \& Education, 50(1):110-124. 
CBIE-LACLO 2015

Anais do XXVI Simpósio Brasileiro de Informática na Educação (SBIE 2015)

Larreamendy-Joerns, J. and Leinhardt, G. (2006). Going the distance with online education. Review of educational research, 76(4):567-605.

Leroy, G., Endicott, J. E., Kauchak, D., Mouradi, O., and Just, M. (2013). User evaluation of the effects of a text simplification algorithm using term familiarity on perception, understanding, learning, and information retention. Journal of medical Internet research, 15(7).

Louden, K. C. (1997). Compiler Construction: Principles and Practice. PWS Publishing Co., Boston, MA, USA.

Luccas, M. R. Z., Chiari, B. M., and Goulart, B. N. G. d. (2012). Reading comprehension of deaf students in regular education. Jornal da Sociedade Brasileira de Fonoaudiologia, 24(4):342-347.

Mangen, A., Walgermo, B. R., and Brønnick, K. (2013). Reading linear texts on paper versus computer screen: Effects on reading comprehension. International Journal of Educational Research, 58:61-68.

Oliveira, F. B. (2012). Desafios na inclusão dos surdos eo intérprete de libras. Revista Diálogos \& Saberes, 8(1).

Quadros, R. M. d. (1997). Educação de surdos: a aquisição da linguagem. Artmed, Porto Alegre, RS, Brasil.

Quadros, R. M. d. (2003). Phrase Structure of Brazilian Sign Language. In Crosslinguistic perspectives in sign language research. Selected papers from TISLR 2000. Signum Press: Hamburg p.141-162.

Quek, F., Elglaly, Y., and Oliveira, F. (2013). Supporting learning for individuals with visual impairment. In Multimedia and Expo Workshops (ICMEW), 2013 IEEE International Conference on, pages 1-6. IEEE.

Santos, G. S., Silveira, M. S., and Aluísio, S. M. (2009). Produção de textos paralelos em língua portuguesa e uma interlíngua de libras. Proceedings of XXXVI Seminário Integrado de Software e Hardware-SEMISH, 9:371-385.

Sassaki, R. K. (2005). Inclusão: o paradigma do século 21. Inclusão: Revista da Educação Especial, 1 (1), pages 19-23.

Schneps, M. H., Thomson, J. M., Chen, C., Sonnert, G., and Pomplun, M. (2013). Ereaders are more effective than paper for some with dyslexia. PloS one, 8(9):e75634.

Siddharthan, A. (2006). Syntactic simplification and text cohesion. Research on Language and Computation, 4(1):77-109.

Silva, I. Q., Matos, P. F., Oliveira, C. F., Leite, T. C., Tavares, K. S., and Silva, B. C. (2014). Avaliação da compreensão de textos jornalísticos em português, em librol e em libras por estudantes surdos. ENCompIF - II Encontro Nacional de Computa $\tilde{\AA} \tilde{\AA} £ o$ dos Institutos Federais.

Torres, E. F., Mazzoni, A. A., and Mello, A. G. d. (2007). Nem toda pessoa cega lê em braille nem toda pessoa surda se comunica em língua de sinais. Educação e Pesquisa, 33(2):369-386. 